\title{
Follow-up of Lesions with Equivocal Radiotracer Uptake on PSMA-Targeted PET in Patients with Prostate Cancer: Predictive Values of the PSMA-RADS-3A and PSMA-RADS-3B Categories
}

\author{
Yafu Yin ${ }^{1-3}$, Rudolf A. Werner ${ }^{1,4}$, Takahiro Higuchi ${ }^{4}$, Constantin Lapa ${ }^{4}$, Kenneth J. Pienta ${ }^{5}$, Martin G. Pomper ${ }^{1,5}$, \\ Michael A. Gorin ${ }^{1,5}$, and Steven P. Rowe ${ }^{1,5}$ \\ ${ }^{I}$ The Russell H. Morgan Department of Radiology and Radiological Science, Johns Hopkins University School of Medicine, \\ Baltimore, Maryland; ${ }^{2}$ Department of Nuclear Medicine, The First Hospital of China Medical University, Shenyang, China; \\ ${ }^{3}$ Department of Nuclear Medicine, Xinhua Hospital, Shanghai Jiao Tong University School of Medicine, Shanghai, China; \\ ${ }^{4}$ Department of Nuclear Medicine, University Hospital Wuerzburg, Wuerzburg, Germany; and ${ }^{5}$ The James Buchanan Brady \\ Urological Institute and Department of Urology, Johns Hopkins University School of Medicine, Baltimore, Maryland
}

Prostate-specific membrane antigen (PSMA)-targeted PET imaging has become commonly used in patients with prostate cancer (PCa). The PSMA reporting and data system version 1.0 (PSMA-RADS version 1.0) categorizes lesions on the basis of the likelihood of $\mathrm{PCa}$ involvement, with PSMA-RADS-3A (soft-tissue) and PSMA-RADS3B (bone) lesions being indeterminate for the presence of disease. We retrospectively reviewed the imaging follow-up of such lesions to determine the rate at which they underwent changes suggestive of underlying PCa. Methods: PET/CT imaging with ${ }^{18} \mathrm{~F}-\mathrm{DCFPyL}$ was performed in 110 patients with $\mathrm{PCa}$, and lesions were categorized according to PSMA-RADS version 1.0. The study reported herein is a retrospective analysis of those patients. Fifty-six of 110 (50.9\%) patients were determined to have indeterminate PSMA-RADS-3A or PSMA-RADS-3B lesions, and 22 of 56 (39.3\%) patients had adequate follow-up to be included in the analysis (median follow-up time was 10 mo [range, 3-22 mo]). The SUV $\mathrm{Sax}_{\max }$ of the lesions was obtained, and the ratios of SUV max of the lesions to $S U V_{\text {mean }}$ of blood pool $\left(\mathrm{SUV}_{\text {max-lesion }} / \mathrm{SUV}_{\text {mean-bloodpool }}\right)$ were calculated. Predetermined criteria were used to evaluate the PSMA-RADS-3A and PSMA-RADS-3B lesions on follow-up imaging to determine whether they demonstrated evidence of underlying malignancy. Results: A total of 46 lesions in 22 patients were considered indeterminate for PCa (i.e., PSMA-RADS-3A [32 lesions] or PSMA-RADS-3B [14 lesions]) and were evaluable on follow-up imaging. Twenty-seven of $46(58.7 \%)$ lesions demonstrated changes suggesting they were true-positive for $\mathrm{PCa}$. These lesions included 24 of $32(75.0 \%)$ PSMA-RADS-3A lesions and 3 of 14 $(21.4 \%)$ lesions categorized as PSMA-RADS-3B. The ranges of $\mathrm{SUV}_{\text {max }}$ and SUV $\mathrm{V}_{\text {max-lesion }} / \mathrm{SUV} \mathrm{V}_{\text {mean-bloodpool }}$ overlapped between those lesions demonstrating changes consistent with malignancy on followup imaging and those lesions that remained unchanged on follow-up. The presence of additional definitive sites of PCa (PSMA-RADS-4 and PSMA-RADS-5) increases the likelihood that indeterminate lesions will manifest as true-positive on follow-up imaging. Conclusion: PSMARADS-3A and PSMA-RADS-3B lesions are truly indeterminate in that

Received Jul. 13, 2018; revision accepted Aug. 28, 2018.

For correspondence or reprints contact: Steven P. Rowe, Division of Nuclear Medicine and Molecular Imaging, The Russell H. Morgan Department of Radiology and Radiological Science, Johns Hopkins University School of Medicine, 601 N. Caroline St., Rm. 3233, Baltimore, MD 21287.

E-mail: srowe8@jhmi.edu

Published online Sep. 6, 2018.

COPYRIGHT (C) 2019 by the Society of Nuclear Medicine and Molecular Imaging. proportions of findings in both categories demonstrate evidence of malignancy on follow-up imaging. Overall, PSMA-RADS-3A lesions are more likely than PSMA-RADS-3B lesions to represent sites of $\mathrm{PCa}$, and this information should be considered when guiding patient therapy.

Key Words: prostate cancer; prostate-specific membrane antigen; PSMA-targeted PET; PSMA-RADS-3A; PSMA-RADS-3B

J Nucl Med 2019; 60:511-516

DOI: 10.2967/jnumed.118.217653

$\mathbf{I}_{n}$ 2018, prostate cancer ( $\mathrm{PCa}$ ) is estimated to be the most commonly diagnosed noncutaneous malignancy as well as the second most common cause of cancer death in U.S. men (1). Despite how common PCa is, imaging of this malignancy has long been challenging, particularly in patients with recurrent or metastatic disease (2). Although conventional imaging can often appropriately stage patients with very advanced disease, it has taken the advent of sensitive molecular imaging agents to be able to reliably identify small-volume disease that may be oligorecurrent/ oligometastatic (3). Such disease may be amenable to metastasisdirected therapy such as salvage lymphadenectomy or stereotactic body radiation therapy $(4,5)$. Those interventions may allow a subset of patients to avoid systemic therapy, and in some cases patients may have prolonged progression-free survival (6).

Among the molecular imaging agents for PCa, PET-based radiotracers that target prostate-specific membrane antigen (PSMA) have shown both exceptional sensitivity and specificity $(7,8)$. Although the improved sensitivity of PSMA-targeted agents relative to conventional imaging has been well established $(2,7,9)$, that superior sensitivity appears to be true even when comparing PSMAtargeted agents with older classes of PCa radiotracers $(10,11)$. This has led to the extensive study of PSMA-targeted compounds in PCa biochemical recurrence $(12,13)$, partially in the hope that detecting sites of recurrent $\mathrm{PCa}$ at low serum prostate-specific antigen (PSA) levels may provide new options for metastasis-directed therapy for carefully selected patients $(14,15)$. Indeed, as the number of publications on PSMA-targeted PET has increased, there has been a 
parallel increase in the number of papers on therapeutic options for oligometastatic PCa (3).

With the implication that findings on PSMA-targeted PET will be used to guide therapy, having a standardized framework that demarcates individual lesions and incorporates information about the imaging specialist's confidence that a lesion represents PCa is valuable. One such system that has been proposed is the PSMA reporting and data system version 1.0 (PSMA-RADS version 1.0) $(16,17)$. PSMA-RADS is predicated on a 5-point scale with PSMARADS-3 indicating an indeterminate lesion. PSMA-RADS-3 can indicate findings, with or without radiotracer uptake, that are unlikely to represent PCa (PSMA-RADS-3C and PSMA-RADS-3D, respectively) $(18,19)$. However, in most cases, indeterminate lesions are those findings that would be typical for PCa such as lymph node (LN) (PSMA-RADS-3A) or bone lesions (PSMA-RADS-3B) and that have low levels of uptake and lack a correlative anatomic finding. In this study, we have longitudinally followed a series of PSMA-RADS-3A and PSMA-RADS-3B lesions to determine how frequently such findings definitively manifest as sites of cancer involvement.

\section{MATERIALS AND METHODS}

\section{Patient Population}

One hundred ten consecutive patients with a history of pathologically diagnosed PCa who had undergone an ${ }^{18} \mathrm{~F}-\mathrm{DCFPyL}$ PET/CT scan on a prospective research protocol (ClinicalTrials.gov NCT02825875) were included in this study. The study reported herein is a retrospective analysis. Patients were imaged under the auspices of a U.S. Food and Drug Administration Investigational New Drug Application (IND 121064). This study was approved by our hospital's Institutional Review Board. All patients signed written, informed consent. Clinical and demographic information including ages, PSA levels, and PCa treatment history were collected.

\section{PET/CT Imaging}

The radiosynthesis of ${ }^{18} \mathrm{~F}$-DCFPyL was performed as has been previously described (20). Images were acquired in a manner consistent with the methods described by Rowe et al. (21). In brief, all patients were asked to refrain from eating or drinking (other than water and medications) for at least $4 \mathrm{~h}$ before the intravenous injection of approximately $333 \mathrm{MBq}(9 \mathrm{mCi})$ of ${ }^{18} \mathrm{~F}$-DCFPyL. Furosemide was not administered. One hour after the injection, whole-body PET/CT was performed (from the midthighs through the vertex of the skull) on either a 128-slice Biograph mCT scanner (Siemens) using time-offlight and reconstructed with a standard iterative reconstruction algorithm provided by the vendor or a 64-slice Discovery RX scanner (GE Healthcare) without time-of-flight and with a standard iterative reconstruction algorithm provided by the vendor. All PET images were passed through a smoothing filter. For the acquisitions, the scanners were in 3-dimensional emission mode with attenuation correction provided by CT. No intravenous or oral CT contrast was administered. On the Siemens scanner, the CT dose was fixed at $40 \mathrm{mAs}$ and on the GE scanner at $80 \mathrm{mAs}$. CT images were reconstructed using standard filtered-backprojection reconstruction algorithms provided by the vendors.

\section{Image Analysis}

${ }^{18} \mathrm{~F}$-DCFPyL PET/CT scans were centrally reviewed by 2 experienced readers, and lesions were categorized according to PSMARADS version 1.0 (16). The reviewers reached a consensus on all lesions included in the analysis. As had previously been set forth in the original PSMA-RADS article (16), the central reviewers considered PSMA-RADS-3A lesions to be those LNs or soft-tissue findings that had subtle radiotracer uptake (approximately blood pool or slightly higher) and that were in a typical pattern of distribution for $\mathrm{PCa}$ (e.g., pelvis and retroperitoneum, as well as mediastinum and left supraclavicular space in patients with more advanced disease (22)). PSMA-RADS-3B lesions could generally be described as sites of lowlevel uptake in the bone without an appreciable anatomic correlate or with punctate sclerosis or other findings on corresponding CT that did not definitively suggest the presence of metastatic disease.

For the patients with lesions categorized with PSMA-RADS-3A or PSMA-RADS-3B, longitudinal follow-up imaging data were sought. Patients were included in further analysis if follow-up imaging at least 3 mo after the baseline ${ }^{18} \mathrm{~F}-\mathrm{DCFPyL}$ PET/CT was available in our institution's PACS. No specific limitations were set on the type of follow-up imaging that could be used, and imaging included repeated ${ }^{18} \mathrm{~F}$-DCFPyL PET/CT, diagnostic CT, or MRI for evaluation of PSMA-RADS-3A lesions and ${ }^{18} \mathrm{~F}-\mathrm{DCFPyL}$ PET/CT, diagnostic CT,

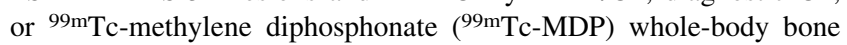
scanning for evaluation of PSMA-RADS-3B lesions.

In addition to PSMA-RADS version 1.0 categorization, the $\mathrm{SUV}_{\max }$ corrected for lean body mass for all of the lesions and the $\mathrm{SUV}_{\text {mean }}$ of blood pool (determined by a $3-\mathrm{cm}$ sphere in the ascending aorta) were measured. The ratios of $\mathrm{SUV}_{\max }$ of each lesion corrected for the $\mathrm{SUV}_{\text {mean }}$ of blood pool $\left(\mathrm{SUV}_{\text {max-lesion }} / \mathrm{SUV}_{\text {mean-bloodpool }}\right)$ were calculated.

In regards to the longitudinal follow-up of the PSMA-RADS-3A and PSMA-RADS-3B lesions, central review was again performed and a consensus was reached as to the nature of the imaging findings on the follow-up studies. Lesions that were determined on follow-up to be suggestive of the presence of PCa met at least one of the following criteria:

1. Follow-up PET/CT imaging with ${ }^{18} \mathrm{~F}-\mathrm{DCFPyL}$ showed uptake of the radiotracer decreased or increased significantly, determined as an $\mathrm{SUV}_{\max }$ change of more than $30 \%$ after therapy (in analogy to PERCIST (23)) OR uptake of the radiotracer increased more than $30 \%$ during observation. This criterion was applied to both PSMARADS-3A and PSMA-RADS-3B lesions.

2. For PSMA-RADS-3A lesions, follow-up CT or MRI showed the diameters of the lesions either decreased or increased more than $2 \mathrm{~mm}$ after therapy OR the diameters of the lesions increased more than 2 mm during observation.

3. For PSMA-RADS-3B lesions, the follow-up CT showed new sclerotic or osteolytic changes $\mathrm{OR}$ baseline faint, indeterminate sclerotic changes demonstrated increased sclerosis.

4. For PSMA-RADS-3B lesions, the follow-up ${ }^{99 \mathrm{~m} T c-M D P}$ wholebody bone scanning showed new avid uptake of radiotracer in the lesions.

\section{Statistical Analysis}

The Fisher exact test was used to compare the number of PSMARADS-3A and PSMA-RADS-3B lesions in different patient groups that were subsequently determined to be true-positive for the presence of malignancy. Descriptive statistics were used to evaluate $\mathrm{SUV}_{\max }$ and $\mathrm{SUV}_{\text {max-lesion }} / \mathrm{SUV}_{\text {mean-bloodpool }}$ in different types of lesions. $P<0.05$ was considered statistically significant for any analysis.

\section{RESULTS}

\section{Patients}

Among the 110 patients, 56 patients (50.9\%) were categorized as having at least 1 PSMA-RADS-3A or PSMA-RADS-3B lesion on ${ }^{18}$ F-DCFPyL PET/CT. However, 34 of $56(60.7 \%)$ lacked adequate imaging follow-up to definitively assess their lesions in a 
longitudinal manner. This relatively high rate of patients without adequate follow-up imaging may have been related to multiple factors including patients undergoing metastasis-directed therapy if their ${ }^{18} \mathrm{~F}$-DCFPyL PET/CT scans showed limited sites of disease and achieving complete biochemical responses or seeking second opinions or transferring care after the results of the scans.

The 22 of 56 (39.3\%) remaining patients were included in the subsequent analysis. The scans had been obtained for biochemical recurrence/persistence in 8 of $22(36.4 \%)$ patients, initial staging in 8 of $22(36.4 \%)$ patients, and evaluation of metastatic disease in 6 of $22(27.3 \%)$ patients. In regards to available follow-up imaging, 20 of $22(90.9 \%)$ patients underwent diagnostic chest/ abdomen/pelvis CT scans, 14 of $22(63.6 \%)$ underwent wholebody bone scanning, 3 of $22(13.6 \%)$ underwent abdomen or pelvis MRI, and 7 of $22(31.8 \%)$ underwent ${ }^{18} \mathrm{~F}$-DCFPyL PET/CT scans. Median follow-up time was 10 mo (range, 3-22 mo). Selected demographic and clinical information on these patients is included in Table 1.

\section{Image Analysis}

Among the 22 patients with usable longitudinal follow-up, there were a total of 46 lesions designated as PSMA-RADS-3A or PSMA-RADS-3B. Thirty-two of $46(69.6 \%)$ were categorized as PSMA-RADS-3A, which included 15 retroperitoneal LNs (15/32, $46.9 \%), 13$ pelvic LNs (13/32, 40.6\%), and 4 supraclavicular LNs $(4 / 32,12.5 \%)$. All included PSMA-RADS-3A LNs measured much less than $1 \mathrm{~cm}$ in axial short-axis diameter (median, $0.3 \mathrm{~cm}$; $\mathrm{SD}, 0.1 \mathrm{~cm}$; range, $0.2-0.5 \mathrm{~cm}$ ). The uptake characteristics of these lesions are summarized in Table 2.

The remaining 14 of $46(30.4 \%)$ lesions were categorized as PSMA-RADS-3B. Of these, 12 of $14(85.7 \%)$ were rib lesions, 1 was a scapula lesion $(1 / 14,7.1 \%)$, and $1(1 / 14,7.1 \%)$ was an iliac bone lesion. The scapula lesion and 2 of the rib lesions were occult on conventional imaging with no anatomic correlates. The iliac bone lesion and 10 of $12(83.3 \%)$ rib lesions demonstrated at least some measure of sclerosis; however, the morphology (either faintly visible or punctate) was determined by the central reviewers to not be definitive for metastatic PCa.

TABLE 1

Clinical and Demographic Data at Time of Baseline ${ }^{18}$ F-DCFPyL PET/CT Scan from Patients Included in This Study

\begin{tabular}{|c|c|c|c|c|}
\hline Patient & Age $(y)$ & Serum PSA $(\mathrm{ng} / \mathrm{mL})$ & Prior PCa Therapy & $\begin{array}{l}\text { PCa Therapy After Baseline } \\
{ }_{18} \text { F-DCFPyL PET/CT }\end{array}$ \\
\hline 1 & 66 & 4.6 & Prostatectomy & Salvage radiation, ADT \\
\hline 2 & 69 & 7.8 & Prostatectomy & Salvage radiation, SBRT, ADT \\
\hline 3 & 56 & 3.9 & $\begin{array}{l}\text { Neoadjuvant Taxotere, neoadjuvant ADT, } \\
\text { prostatectomy }\end{array}$ & SBRT \\
\hline 4 & 66 & 6.48 & $\begin{array}{l}\text { Prostatectomy, salvage radiation, ADT, } \\
\text { SBRT, provenge, }{ }^{177} \text { Lu-PSMA }\end{array}$ & Taxotere \\
\hline 5 & 68 & 22.3 & $\begin{array}{l}\text { Prostatectomy, salvage radiation, } \\
\text { investigational DNA-based vaccine }\end{array}$ & ADT \\
\hline 6 & 63 & 12 & Prostatectomy, ADT & ABRT, Provenge, Enzalutamide \\
\hline 7 & 66 & 0.4 & Prostatectomy, salvage radiation, ADT & None \\
\hline 8 & 65 & 0.5 & Taxotere, ADT, SBRT, Provenge & Provenge, SBRT, ${ }^{177}$ Lu-PSMA \\
\hline 9 & 61 & 0.3 & Prostatectomy & Salvage radiation \\
\hline 10 & 65 & 37.8 & None & EBRT, ADT \\
\hline 11 & 71 & 10.8 & None & Prostatectomy \\
\hline 12 & 63 & 2.2 & Prostatectomy, ADT & ADT \\
\hline 13 & 59 & 5.8 & Prostatectomy, salvage radiation, ADT & SBRT \\
\hline 14 & 64 & 34.3 & $\begin{array}{l}\text { Prostatectomy, salvage radiation, ADT, } \\
\text { investigational DNA-based vaccine }\end{array}$ & ADT \\
\hline 15 & 53 & 23.3 & None & Taxotere, ADT \\
\hline 16 & 64 & 5.6 & $\begin{array}{l}\text { Prostatectomy, salvage radiation, ADT, } \\
{ }^{177} \text { Lu-PSMA, Provenge, Enzalutamide }\end{array}$ & Abiraterone \\
\hline 17 & 69 & 1.4 & Prostatectomy, salvage radiation & ADT \\
\hline 18 & 50 & 0.7 & Prostatectomy & Taxotere, ADT \\
\hline 19 & 64 & 70.4 & None & Taxotere, ADT \\
\hline 20 & 54 & 21.4 & None & Prostatectomy, Abiraterone, ADT \\
\hline 21 & 69 & 5.3 & $\begin{array}{l}\text { Prostatectomy, salvage radiation, ADT, } \\
{ }^{177} \text { Lu-PSMA, SBRT }\end{array}$ & ADT, Carboplatin, Etoposide \\
\hline 22 & 63 & 9.7 & None & ADT \\
\hline
\end{tabular}

$\mathrm{ADT}=$ androgen deprivation therapy; SBRT = stereotactic body radiation therapy; EBRT = external beam radiation therapy. 
TABLE 2

Characteristics of PSMA-RADS-3A and PSMA-RADS-3B Lesions

\begin{tabular}{lrrrrrr}
\hline $\begin{array}{l}\text { PSMA-RADS } \\
\text { score }\end{array}$ & $\begin{array}{c}\text { No. of } \\
\text { lesions }\end{array}$ & $\begin{array}{c}\text { Median } \\
\text { SUV }_{\text {max }}\end{array}$ & $\begin{array}{c}\text { No. of lesions with } \\
\text { imaging changes suggesting } \\
\text { malignancy at follow-up }\end{array}$ & $\begin{array}{c}\text { Median SUV } \\
\text { of changed lesions }\end{array}$ & $\begin{array}{c}\text { No. of unchanged lesions on follow-up unchanged lesions } \\
\text { Median SUV }\end{array}$ \\
\hline 3A & 32 & $1.62(0.93-2.32)$ & $24(75.0 \%)$ & $1.59(1.05-2.32)$ & $8(25.0 \%)$ & $1.62(0.93-2.09)$ \\
3B & 14 & $1.15(0.85-1.89)$ & $3(21.4 \%)$ & $1.35(1.23-1.89)$ & $11(78.6 \%)$ & $1.00(0.85-1.54)$ \\
Total & 46 & $1.44(0.85-2.32)$ & $27(58.7 \%)$ & $1.53(1.05-2.32)$ & $19(41.3 \%)$ & $1.30(0.85-2.09)$ \\
\hline
\end{tabular}

In total, 27 of 46 (58.7\%) PSMA-RADS-3A and PSMA-RADS3B lesions demonstrated changes on follow-up imaging suggesting that they were true-positive for PCa involvement (Table 2). Among those 27 lesions, 13 of $27(48.1 \%)$ met criteria 1 and 2 (from the "Materials and Methods" section), 9 of 27 (33.3\%) met criterion 2, 2 of $27(7.4 \%)$ met criterion 1, 1 of $27(3.7 \%)$ met criteria 1 and 3,1 of $27(3.7 \%)$ met criterion 3, and 1 of $27(3.7 \%)$ met criteria 3 and 4 .

In regards to PSMA-RADS-3A lesions, 24 of 32 (75.0\%) demonstrated changes on follow-up imaging consistent with the lesions being true-positive for PCa involvement (Fig. 1). In contradistinction, for PSMA-RADS-3B lesions, 3 of 14 (21.4\%) lesions had findings on follow-up imaging appearing to confirm true positivity for PCa involvement (Fig. 2). On a patient-level analysis, 15 of 22 patients $(68.2 \%)$ had at least 1 PSMA-RADS-3A or PSMA-RADS-3B lesion undergo changes on follow-up imaging consistent with malignant involvement. In terms of number of lesions per patient, 13 of $22(59.1 \%)$ patients had a single PSMARADS-3A or PSMA-RADS-3B lesion and 9 of 22 (40.9\%) patients had 2 or more such lesions (maximum 7). Nine of 13 (69.2\%) solitary lesions had findings on follow-up imaging consistent with disease involvement. For the patients with more than one PSMA-RADS-3A or PSMA-RADS-3B finding, 18 of $33(54.5 \%)$ lesions in 6 of 9 $(66.7 \%)$ patients demonstrated evidence of malignancy on followup imaging.
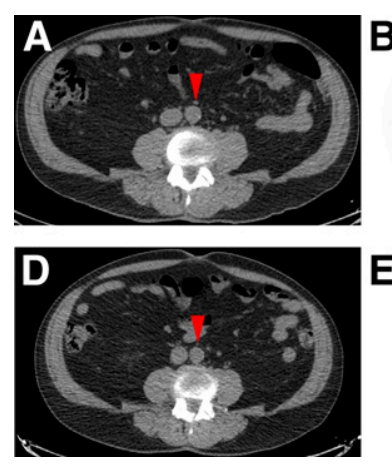
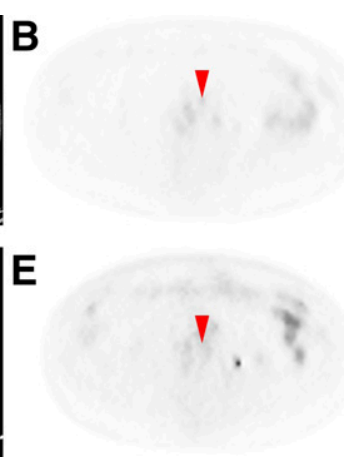
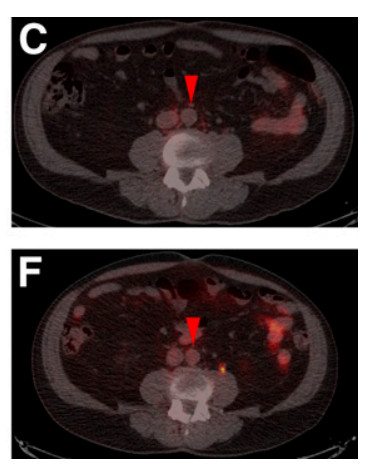

FIGURE 1. Axial attenuation correction CT (A), axial ${ }^{18} \mathrm{~F}-\mathrm{DCFPyL}$ PET (B), and axial ${ }^{18} \mathrm{~F}-\mathrm{DCFPyL}$ PET/CT (C) from a patient with a 3-mm short-axis, preaortic, retroperitoneal LN with faint radiotracer uptake slightly higher than blood pool (red arrowheads). This was determined by central review to be a PSMA-RADS-3A lesion. Follow-up axial attenuation-correction CT (D), axial ${ }^{18} \mathrm{~F}$ DCFPyL PET (E), and axial ${ }^{18} \mathrm{~F}-\mathrm{DCFPyL}$ PET/CT (F) 6 mo later after multiple treatment modalities including androgen deprivation therapy. The node had definitively decreased in size and lacked any discernable uptake on the follow-up scan (red arrowheads), most compatible with PCa involvement.
Stratifying by the number of PSMA-RADS-4 and PSMA-RADS5 lesions present in the same patient on baseline ${ }^{18} \mathrm{~F}$-DCFPyL PET/ CT indicated that PSMA-RADS-3A and PSMA-RADS-3B findings were more likely to evidence signs of malignancy on follow-up imaging if other definitively malignant lesions were present. The lesions were divided into 3 groups: group $1(n=10)$ without PSMA-RADS-4 (i.e., PCa highly likely) or PSMA-RADS-5 (i.e., $\mathrm{PCa}$ almost certainly present) lesions in the same patient, group 2 ( $n=16$ ) with 1-3 PSMA-RADS-4 or PSMA-RADS-5 lesions in the same patient, and group $3(n=20)$ with more than 3 PSMARADS-4 and PSMA-RADS-5 lesions in the same patient. The number of lesions demonstrating evidence of malignancy on follow-up imaging was 3 of $10(30.0 \%)$ in group 1, 6 of $16(37.5 \%)$ in group 2, oups reached statistical significance $(P<0.05)$.

SU $V_{\max } \mathrm{S}$ of PSMA-RADS-3A and PSMA-RADS-3B leSUV $_{\text {max }}$ of PSMA-RADS-3A lesions was 1.62 , with a range from 0.93 to 2.32 , whereas the median SUV $_{\max }$ of PSMA-RADS-3B lesions was 1.15 , with a range from 0.85 to 1.89 , respectively) (Table 2). In comparing those lesions that had characteristic changes of malignancy on follow-up imaging versus those that remained unchanged, the median $\mathrm{SUV}_{\max }$ was actually marginally higher for the unchanged lesions, although again the $\mathrm{SUV}_{\max }$ ranges overlapped (median $\mathrm{SUV}_{\max }$ for changed lesions was 1.53, with a range from 1.05 to 2.32 , whereas the median $\mathrm{SUV}_{\max }$ for unchanged lesions was 1.30 with a range from 0.85 to 2.09 , respectively) (Table 2). Additional characteristics of the lesions can be found in Table 2.

The analyses based on $\mathrm{SUV}_{\text {max-lesion }}$ / $\mathrm{SUV}_{\text {mean-bloodpool }}$ produced similar results, suggesting that the $\mathrm{SUV}_{\max }$ ranges encountered for PSMA-RADS-3A and PSMARADS-3B lesions are not significantly impacted by blood-pool radiotracer concentration at the time of imaging. The median $\mathrm{SUV}_{\text {max-lesion }} / \mathrm{SUV}_{\text {mean-bloodpool }}$ for PSMARADS-3A lesions was 1.64 , with a range from 0.90 to 2.81 , and for PSMA-RADS$3 \mathrm{~B}$ lesions the median was 1.18 , with a range from 0.77 to 1.63 . Again, the ranges of $\mathrm{SUV}_{\text {max-lesion }} / \mathrm{SUV}_{\text {mean-bloodpool }}$ for lesions 


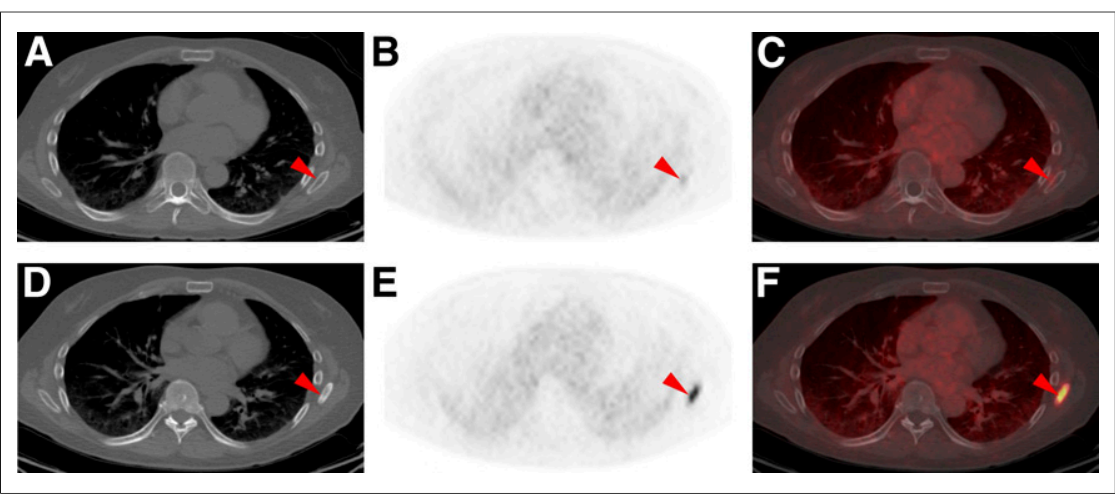

FIGURE 2. Axial attenuation-correction CT (A), axial ${ }^{18} \mathrm{~F}$-DCFPyL PET (B), and axial ${ }^{18} \mathrm{~F}-\mathrm{DCFPyL}$ $\mathrm{PET} / \mathrm{CT}$ (C) from a patient with faint uptake in inferior right scapula (red arrowheads). This was determined on central review to represent a PSMA-RADS-3B lesion without a visible anatomic correlate. Follow-up axial attenuation correction CT (D), axial ${ }^{18} \mathrm{~F}-\mathrm{DCFPyL}$ PET (E), and axial ${ }^{18} \mathrm{~F}-$ DCFPyL PET/CT (F) 10 mo later show markedly increased radiotracer uptake and new sclerosis in inferior right scapula (red arrowheads), most consistent with a PCa bone metastasis. Patient had started on taxotere chemotherapy but had a rising PSA level at time of follow-up imaging, consistent with progressive systemic disease.

with changes suggesting malignancy versus unchanged lesions overlapped (median $\mathrm{SUV}_{\text {max-lesion }} / \mathrm{SUV}_{\text {mean-bloodpool }}$ for changed PSMARADS-3A lesions was 1.65 [range, 0.90-2.81], median SUV $_{\text {max-lesion }}$ ' SUV $_{\text {mean-bloodpool }}$ for unchanged PSMA-RADS-3A lesions was 1.57 [range, 1.18-1.76], median $\mathrm{SUV}_{\text {max-lesion }} / \mathrm{SUV}_{\text {mean-bloodpool }}$ for changed PSMA-RADS-3B lesions was 1.23 [range, 1.04-1.42], and median $\mathrm{SUV}_{\text {max-lesion }} / \mathrm{SUV}_{\text {mean-bloodpool }}$ for unchanged PSMA-RADS3B lesions was 1.16 [range, 0.77 to 1.63 ]).

\section{DISCUSSION}

PSMA-targeted PET has been rapidly adopted around the world for PCa imaging given its high sensitivity and specificity for the identification of sites of disease $(8,24)$. However, as with any imaging modality, there are indeterminate findings that arise either as a result of incidental findings or because of lesion imaging characteristics that belie easy categorization (18). The preponderance of studies on PSMA-targeted PET imaging have been retrospective, further hindering the ability of readers to know how to handle indeterminate findings, although large prospective studies are ongoing (25). As a result, multiple systems have been proposed to add structure to the interpretation of PSMA-targeted PET scans $(16,26,27)$. Although these systems emphasize different aspects of PSMA-targeted PET scan interpretation, the central aspect of PSMA-RADS version 1.0 is the categorization of lesions based on the interpreting imaging specialist's suspicion of the presence of PCa $(16,17)$. This aligns PSMA-RADS with previously reported organ-based reporting and data systems such as those for breast (breast imaging reporting and data system, BIRADS (28)) and multiparametric prostate MRI (prostate imaging reporting and data system, PI-RADS (29)). For example, BIRADS includes a total of 7 categories that overall represent different probabilities of imaging findings being malignant and proffer corresponding recommendations (e.g., BI-RADS 3 indicates the need for 6-mo follow-up imaging in the context of a less than $2 \%$ chance of the finding being malignant, whereas BI-RADS 4 lesions will be found to be cancer at a rate of approximately $30 \%$ and BI-RADS 5 lesions are almost certainly cancer with a positive predictive value of about $97 \%(28)$ ). Knowledge of the BI-RADS categories is useful for both radiologists and clinicians for communication and guidance of patient management (30).

The potential adoption of PSMA-RADS is contingent on a similar utility for guiding clinical decision making. On a global level, nearly $60 \%$ of indeterminate lesions on PSMA-targeted ${ }^{18} \mathrm{~F}$-DCFPyL PET/CT were found to have changes on follow-up imaging compatible with disease involvement, with $75.0 \%$ of PSMA-RADS-3A LN lesions and $21.4 \%$ of PSMA-RADS-3B bone lesions meeting our prespecified criteria for harboring $\mathrm{PCa}$. The presence of more definitive sites of PCa (i.e., PSMARADS-4 and PSMA-RADS-5 lesions) increased the likelihood of PSMA-RADS-3A and PSMA-RADS-3B findings having follow-up imaging findings consistent with the presence of PCa. The ranges of SUVs between those indeterminate findings that showed evidence of malignancy on follow-up imaging and those that remained stable overlapped, suggesting that a simple difference in uptake cannot be used to distinguish between those 2 types of lesions.

These findings bear out that PSMA-RADS-3A and PSMARADS-3B are truly indeterminate lesions. Thus, depending on the clinical context, image-guided biopsy or follow-up imaging are required to determine the likelihood of malignancy. In particular, follow-up imaging may be of particular value in isolated PSMARADS-3A lesions when there are no other findings on the scan. PSMA-RADS-3B lesions warrant careful consideration as only a minority of such findings will have characteristic changes of $\mathrm{PCa}$ involvement on follow-up imaging. If patients are considered for salvage or focal therapies as opposed to systemic therapy, it will be necessary for clinicians to weigh the potential cost and toxicities associated with those therapies against the likelihood of PSMA-RADS-3A and PSMA-RADS-3B lesions representing true sites of disease. Although PSMA-RADS was developed primarily on the basis of experience with the ${ }^{18} \mathrm{~F}$-labeled radiotracer ${ }^{18}$ F-DCFPyL, the overall similarities of mechanisms and biodistributions of other PSMA-targeted, urea-based, small-molecule radiotracers leads us to believe that this system would be applicable with any of those other agents and that similar conclusions could be drawn regarding follow-up of indeterminate lesions.

The most significant limitations to the current study are its retrospective nature and that lesions were not correlated to histopathology. As would be expected for indeterminate lesions on PSMA-targeted PET imaging, the findings were often small and would be difficult to reliably target with conventional imaging guidance for biopsy. Therefore, histopathology is a difficult gold standard to apply to this study, and follow-up imaging findings may represent a more practical approach to determining the nature of lesions (2). Further, because of the small size or lack of conspicuity of many PSMA-RADS-3A and PSMA-RADS-3B lesions on conventional imaging, we were unable to apply commonly used response/progression criteria such as RECIST 1.1 (31) to objectively categorize findings on follow-up. We also acknowledge that with the often indolent nature of PCa, some lesions that remained unchanged on follow-up imaging could still represent sites of PCa and that our percentages of PSMA-RADS-3A and PSMA-RADS-3B 
lesions that we considered consistent with PCa involvement may be underestimated. Prospective longitudinal follow-up of a larger number of indeterminate lesions from PSMA-targeted PET scans will be critical to address these limitations. Lastly, PSMA-RADS version 1.0 is a construct that was derived from a single center experience, and several additional measures of validation, as well as validation at other sites, is necessary to establish if widespread adoption of this system is warranted.

\section{CONCLUSION}

In this study of longitudinal follow-up of lesions with indeterminate levels of uptake on PSMA-targeted PET, a significant majority (75.0\%) of PSMA-RADS-3A lesions demonstrated changes on subsequent imaging compatible with the presence of PCa. However, only a minority $(21.4 \%)$ of PSMA-RADS-3B indeterminate bone lesions showed changes on follow-up imaging suggestive of underlying PCa. The presence of additional definitive sites of $\mathrm{PCa}$ increases the likelihood that indeterminate lesions will manifest as true-positive on follow-up. Of note, $\mathrm{SUV}_{\max }$ does not appear to be a useful discriminator to assess the true nature of indeterminate lesions. These findings confirm the necessity for a category in the PSMA-RADS grading system for indeterminate lesions.

\section{DISCLOSURE}

We gratefully acknowledge funding from the Prostate Cancer Foundation Young Investigator Award and National Institutes of Health grants CA134675, CA184228, EB024495, and CA183031. This project has received funding from the European Union's Horizon 2020 research and innovation programme under the Marie Sklodowska-Curie grant agreement no. 701983. Michael A. Gorin is a coinventor on a U.S. patent covering ${ }^{18} \mathrm{~F}-\mathrm{DCFPyL}$ and as such is entitled to a portion of any licensing fees and royalties generated by this technology. This arrangement has been reviewed and approved by the Johns Hopkins University in accordance with its conflict of interest policies. Michael A. Gorin has served as a consultant for Progenics Pharmaceuticals, Inc., the licensee of ${ }^{18} \mathrm{~F}$ DCFPyL. All authors have received research funding from Progenics Pharmaceuticals, Inc. No other potential conflict of interest relevant to this article was reported.

\section{REFERENCES}

1. Siegel RL, Miller KD, Jemal A. Cancer statistics, 2018. CA Cancer J Clin. 2018;68:7-30.

2. Rowe SP, Macura KJ, Ciarallo A, et al. Comparison of prostate-specific membrane antigen-based ${ }^{18} \mathrm{~F}$-DCFBC PET/CT to conventional imaging modalities for detection of hormone-naive and castration-resistant metastatic prostate cancer. J Nucl Med. 2016;57:46-53.

3. Murphy DG, Sweeney CJ, Tombal B. "Gotta catch 'em all", or do we? Pokemet approach to metastatic prostate cancer. Eur Urol. 2017;72:1-3.

4. Siriwardana A, Thompson J, van Leeuwen PJ, et al. Initial multicentre experience of ${ }^{68}$ gallium-PSMA PET/CT guided robot-assisted salvage lymphadenectomy: acceptable safety profile but oncological benefit appears limited. BJU Int. 2017;120:673-681.

5. Henkenberens $\mathrm{C}$, von Klot CA, Ross TL, et al. ${ }^{68} \mathrm{Ga}$-PSMA ligand PET/CT-based radiotherapy in locally recurrent and recurrent oligometastatic prostate cancer: early efficacy after primary therapy. Strahlenther Onkol. 2016;192:431-439.

6. Tosoian JJ, Gorin MA, Ross AE, Pienta KJ, Tran PT, Schaeffer EM. Oligometastatic prostate cancer: definitions, clinical outcomes, and treatment considerations. Nat Rev Urol. 2017;14:15-25.

7. Rowe SP, Macura KJ, Mena E, et al. PSMA-based $\left[{ }^{18} \mathrm{~F}\right] \mathrm{DCFPyL}$ PET/CT is superior to conventional imaging for lesion detection in patients with metastatic prostate cancer. Mol Imaging Biol. 2016;18:411-419.

8. Perera M, Papa N, Christidis D, et al. Sensitivity, specificity, and predictors of positive ${ }^{68} \mathrm{Ga}$-prostate-specific membrane antigen positron emission tomography in advanced prostate cancer: a systematic review and meta-analysis. Eur Urol. 2016;70:926-937.

9. Rowe SP, Mana-Ay M, Javadi MS, et al. PSMA-based detection of prostate cancer bone lesions with ${ }^{18} \mathrm{~F}-\mathrm{DCFPyL}$ PET/CT: a sensitive alternative to ${ }^{99 \mathrm{~m}} \mathrm{Tc}-\mathrm{MDP}$ bone scan and $\mathrm{Na}^{18} \mathrm{~F}$ PET/CT? Clin Genitourin Cancer. 2016;14:e115-e118.

10. Afshar-Oromieh A, Zechmann CM, Malcher A, et al. Comparison of PET imaging with a ${ }^{68} \mathrm{Ga}$-labelled PSMA ligand and ${ }^{18} \mathrm{~F}$-choline-based PET/CT for the diagnosis of recurrent prostate cancer. Eur J Nucl Med Mol Imaging. 2014;41: 11-20.

11. Morigi JJ, Stricker PD, van Leeuwen PJ, et al. Prospective comparison of ${ }^{18} \mathrm{~F}$ fluoromethylcholine versus ${ }^{68} \mathrm{Ga}$-PSMA PET/CT in prostate cancer patients who have rising psa after curative treatment and are being considered for targeted therapy. J Nucl Med. 2015;56:1185-1190.

12. Eiber M, Maurer T, Souvatzoglou M, et al. Evaluation of hybrid ${ }^{68} \mathrm{Ga}-\mathrm{PSMA}$ ligand PET/CT in 248 patients with biochemical recurrence after radical prostatectomy. J Nucl Med. 2015;56:668-674.

13. Afshar-Oromieh A, Holland-Letz T, Giesel FL, et al. Diagnostic performance of ${ }^{68} \mathrm{Ga}$-PSMA-11 (HBED-CC) PET/CT in patients with recurrent prostate cancer: evaluation in 1007 patients. Eur J Nucl Med Mol Imaging. 2017;44:12581268.

14. Siva S, Bressel M, Murphy DG, et al. Stereotactic ablative body radiotherapy (SABR) for oligometastatic prostate cancer: a prospective clinical trial. Eur Urol. 2018;74:455-462.

15. Ost P, Reynders D, Decaestecker K, et al. Surveillance or metastasis-directed therapy for oligometastatic prostate cancer recurrence: a prospective, randomized, multicenter phase II trial. J Clin Oncol. 2018;36:446-453.

16. Rowe SP, Pienta KJ, Pomper MG, Gorin MA. Proposal for a structured reporting system for prostate-specific membrane antigen-targeted pet imaging: PSMARADS version 1.0. J Nucl Med. 2018;59:479-485.

17. Rowe SP, Pienta KJ, Pomper MG, Gorin MA. PSMA-RADS version 1.0: a step towards standardizing the interpretation and reporting of PSMA-targeted PET imaging studies. Eur Urol. 2018;73:485-487.

18. Sheikhbahaei S, Afshar-Oromieh A, Eiber M, et al. Pearls and pitfalls in clinical interpretation of prostate-specific membrane antigen (PSMA)-targeted PET imaging. Eur J Nucl Med Mol Imaging. 2017;44:2117-2136.

19. Salas Fragomeni RA, Amir T, Sheikhbahaei S, et al. Imaging of nonprostate cancers using PSMA-targeted radiotracers: rationale, current state of the field, and a call to arms. J Nucl Med. 2018;59:871-877.

20. Ravert HT, Holt DP, Chen Y, et al. An improved synthesis of the radiolabeled prostate-specific membrane antigen inhibitor, $\left[{ }^{18} \mathrm{~F}\right] \mathrm{DCFPyL} . J$ Labelled Comp Radiopharm. 2016;59:439-450.

21. Rowe SP, Gorin MA, Hammers HJ, et al. Imaging of metastatic clear cell renal cell carcinoma with PSMA-targeted ${ }^{18}$ F-DCFPyL PET/CT. Ann Nucl Med. 2015;29:877-882.

22. Werner RA, Andree C, Javadi MS, et al. A voice from the past: rediscovering the virchow node with prostate-specific membrane antigen-targeted ${ }^{18} \mathrm{~F}-\mathrm{DCFPyL}$ positron emission tomography imaging. Urology. 2018;117:18-21.

23. Wahl RL, Jacene H, Kasamon Y, Lodge MA. From RECIST to PERCIST: evolving considerations for PET response criteria in solid tumors. J Nucl Med. 2009;50(suppl 1):122S-150S

24. Murphy DG, Hofman M, Lawrentschuk N, Maurer T. Bringing clarity or confusion? The role of prostate-specific membrane antigen positron-emission/ computed tomography for primary staging in prostate cancer. BJU Int. 2017;119: 194-195.

25. Hofman MS, Murphy DG, Williams SG, et al. A prospective randomized multicentre study of the impact of gallium- 68 prostate-specific membrane antigen (PSMA) PET/CT imaging for staging high-risk prostate cancer prior to curative-intent surgery or radiotherapy (proPSMA study): clinical trial protocol. $B J U$ Int. 2018;122:783-793.

26. Fanti S, Minozzi S, Morigi JJ, et al. Development of standardized image interpretation for ${ }^{68} \mathrm{Ga}$-PSMA PET/CT to detect prostate cancer recurrent lesions. Eur J Nucl Med Mol Imaging. 2017;44:1622-1635.

27. Eiber M, Herrmann K, Calais J, et al. Prostate Cancer Molecular Imaging Standardized Evaluation (PROMISE): proposed miTNM classification for the interpretation of PSMA-ligand PET/CT. J Nucl Med. 2018;59:469-478.

28. Orel SG, Kay N, Reynolds C, Sullivan DC. BI-RADS categorization as a predictor of malignancy. Radiology. 1999;211:845-850.

29. Weinreb JC, Barentsz JO, Choyke PL, et al. PI-RADS Prostate Imaging-Reporting and Data System: 2015, version 2. Eur Urol. 2016;69:16-40.

30. Balleyguier C, Ayadi S, Van Nguyen K, Vanel D, Dromain C, Sigal R. BIRADS classification in mammography. Eur J Radiol. 2007;61:192-194.

31. Eisenhauer EA, Therasse P, Bogaerts J, et al. New response evaluation criteria in solid tumours: revised RECIST guideline (version 1.1). Eur J Cancer. 2009;45: 228-247. 